\title{
Acoustic signal characteristics of laser induced cavitation in DDFP droplet: Spectrum and time-frequency analysis
}

\author{
Yi Feng*, Dui Qin, Jun Zhang, Chenxiang Ma and Mingxi Wan \\ The Key Laboratory of Biomedical Information Engineering of Ministry of Education, Department of \\ Biomedical Engineering, School of Life Science and Technology, Xi'an Jiaotong University, Xi'an, \\ 710049, P.R. China
}

\begin{abstract}
Cavitation has great application potential in microvessel damage and targeted drug delivery. Concerning cavitation, droplet vaporization has been widely investigated in vitro and in vivo with plasmonic nanoparticles. Droplets with a liquid dodecafluoropentane (DDFP) core enclosed in an albumin shell have a stable and simple structure with good characteristics of laser absorbing; thus, DDFP droplets could be an effective aim for laser-induced cavitation. The DDPF droplet was prepared and perfused in a mimic microvessel in the optical microscopic system with a passive acoustic detection module. Three patterns of laser-induced cavitation in the droplets were observed. The emitted acoustic signals showed specific spectrum components at specific time points. It was suggested that a nanosecond laser pulse could induce cavitation in DDPF droplets, and specific acoustic signals would be emitted. Analyzing its characteristics could aid in monitoring the laserinduced cavitation process in droplets, which is meaningful to theranostic application.
\end{abstract}

Keywords: DDFP droplet, laser induced cavitation, acoustic signal, spectrum analysis, time-frequency analysis

\section{Introduction}

Cavitation is an effective methodology to induce microvessel damage and then aid in inhibiting tumor growth or improving the efficiency of drug delivery [1]. Furthermore, a feasible means of generating this particular damage is through cavitation that is induced by droplet vaporization [2], which is a process that is initiated with the aid of plasmonic nanoparticles that absorb the energy from a laser and result in the instantaneous heating of the particles [3]. However, these particular plasmonic nanoparticles have a complicated structure and are expensive [4]. In this paper, dodecafluoropentane (DDFP) droplets, which are easily fabricated, were induced to cavitation by a nanosecond laser pulse with low energy in an in vitro experiment. The patterns of laser-induced cavitation in the droplet were observed by high-speed microscopic photography. Moreover, the emitted acoustic signal was acquired by passive cavitation detection and then was analyzed. It revealed that the DDFP droplets absorb the

\footnotetext{
* Address for correspondence: Yi Feng, The Key Laboratory of Biomedical Information Engineering of Ministry of Education, Department of Biomedical Engineering, School of Life Science and Technology, Xi'an Jiaotong University, Xi'an, 710049, P.R. China. Tel.: +862982668668-805; Fax:+862982668668; Email: fengyi@mail.xjtu.edu.cn.
} 
energy well and cause the laser-induced cavitation, and specific acoustic signals are emitted during the process. The acoustic characteristics of laser-induced cavitation can be used to monitor the cavitation process, which is significant for determining its application in theranostics, such as microvesselcontrolled damage, for example.

\section{Material and method}

\subsection{Preparation of DDFP droplets}

The droplets of liquid dodecafluoropentane (DDFP, $\mathrm{C}_{5} \mathrm{~F}_{12}$, boiling point of $29^{\circ} \mathrm{C}$, Sigma, St. Louis, MO, USA) enclosed in an albumin shell (bovine serum albumin, BSA, Sigma, St. Louis, MO, USA) were prepared as previously described in an earlier literature [5]. First, $0.3 \mathrm{~mL}$ of DDFP and $9.7 \mathrm{~mL}$ of degassed deionized water were mixed and emulsified with an ultrasonic liquid processor (VCX 750, Sonics \& Materials Inc, USA) for $30 \mathrm{~s}$. Then, the resulting emulsion was poured slowly into a 5-mL albumin solution $(20 \mathrm{mg} / \mathrm{mL})$ to coat the droplet with an albumin shell, which was used to stabilize the emulsion by lowering the surface tension of the droplets as well as inhibiting coalescence. After coating, the mixture was filtered through a disposable syringe filter $(450 \mathrm{~nm}$ pore size, polyvinylidene difluoride membrane, Whatman, Inc., Pisataway, NJ, USA). Although the boiling point of DDFP in bulk is $29^{\circ} \mathrm{C}$, it has been reported that DDFP in the form of nanodroplets has a higher boiling point of at least $74^{\circ} \mathrm{C}$ [6]. Furthermore, the size distribution of the DDFP droplets was examined by a laser particle size analyzer (Nano-NS90, Malvern Instruments Ltd., UK).

\subsection{Laser irradiation and acoustic detection system}

The experimental system (Figure 1) included the following: a laser generator (Andor Technology Ltd., Northern Ireland), a focusing optics system based on long-distance microscopy (TiU, Nikon, Nikon Instruments Inc., Japan) with $40 \times$ and 0.8 numerical aperture objective lens, one high speed camera, a passive acoustic detection system with a broadband receiver and a high speed digitizer, a

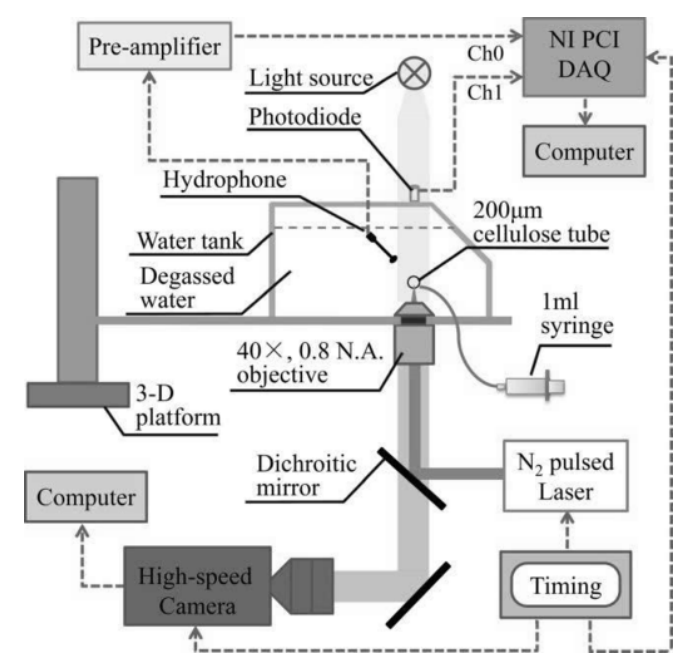

Fig. 1. Experimental system. 
sufficient light source, and a timing system. Two foci of laser and the acoustic detection system were confocal on a $200 \mu \mathrm{m}$ cellulose tube (Spectra Por, Spectrum Laboratories Inc., USA) that was filled with DDFP droplets. Moreover, to ensure synchronization of the laser pulse, flash illumination, and high-speed camera operation, each component was electronically triggered with appropriately delayed TTL pulses from the timing system. In addition, a single pulse laser of $521 \mathrm{~nm}$ wavelength with a 3-5 ns pulse width and an average power of $50 \mu \mathrm{J}$ was employed in the experiment. To verify the time at which the cavitation formed, a photodiode (AEPX35, Centronics, USA) was introduced to mark the time at which the laser induces cavitation in the DDFP droplet. The entire system was constructed and kept on a self-leveling and vibration damping optical table.

\subsection{High speed optical observation}

The laser-induced cavitation process in the droplets was observed optically using a long-distance inverted microscope system. An unfocused white light source illuminated the sample from above, and through the same objective that was used to focus the laser beam, a scattered light from the bubble was projected onto a high-speed camera. The resulting images were recorded in a digital image format with an adjustable resolution and frame rate. Furthermore, selecting a lower resolution allowed for a higher maximum frame rate.

In the results presented below, $t=0 \mu s$ is defined as the temporal closest frame at the instant at which the laser pulse is incident to the DDFP droplet. The high-speed camera operation was internally set to $t$ $=-0.1 \mathrm{~s}$ so as to capture a number of frames prior to the initiation of the cavitation activity.

\subsection{Acoustic detection and signal analysis}

The cavitation process was also detected acoustically in a passive mode with a needle hydrophone ( $0.5 \mathrm{~mm}$ diameter, Precision Acoustics Ltd., Dorchester, UK). Furthermore, a broadband receiver (BR-640, Ritec, USA) and a high-speed digitizer (PCI-5122, National Instruments, USA) were used to amplify and ultimately record the acoustic signal acquired by the hydrophone. The recorded acoustic signals were stored in the DAT format for subsequent signal processing via the MATLAB software (The Mathworks Inc., Natick, MA).

In addition, the recorded ultrasound radio frequency (RF) signal was first read and filtered through a band-pass filter. Then, spectral analysis was performed in order to obtain the characteristics of the laser-induced cavitation in the DDFP droplets through a Fast Fourier Transformation (FFT) of the waveform. In order to acquire a more intuitive understanding of the changes to the cavitation spectrum over time, the joint time-frequency analysis (JTFA) with an adaptive optimal kernel time-frequency analysis tool (AOK-TFR) was applied to further analyze the acoustic signals that were generated during the cavitation process. Compared to the short-time FFT transform, which possesses a higher resolution in the frequency domain, the adaptive optimal kernel time-frequency analysis tool has a higher resolution in the time domain.

\section{Results and discussion}

\subsection{Patterns of laser-induced cavitation in DDFP droplets}

There are three patterns of laser-induced cavitation in DDFP droplets, as are shown in Figure 2. In 


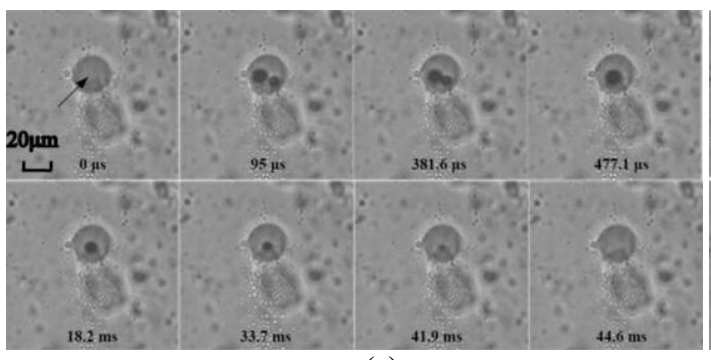

(a)

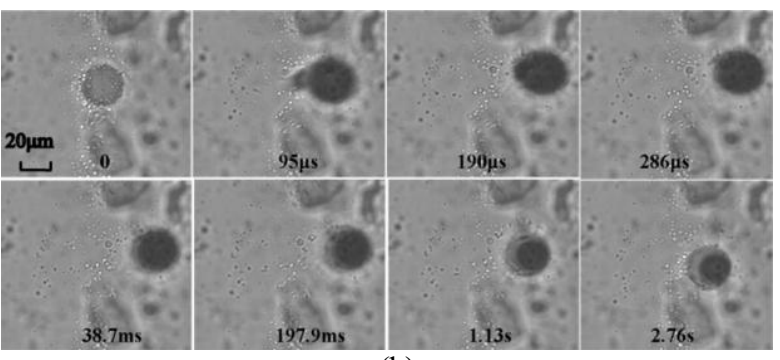

(b)

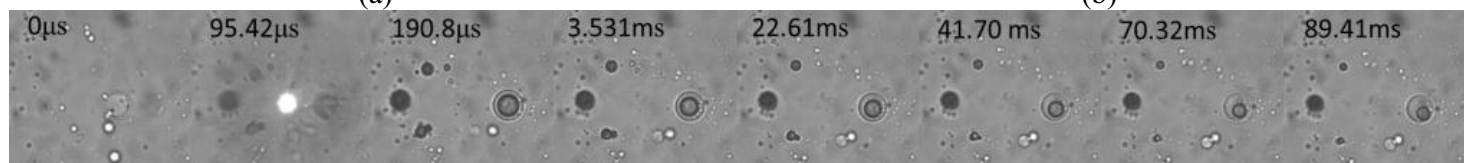

(c)

Fig. 2. Three patterns of laser induced cavitation in DDFP droplet.

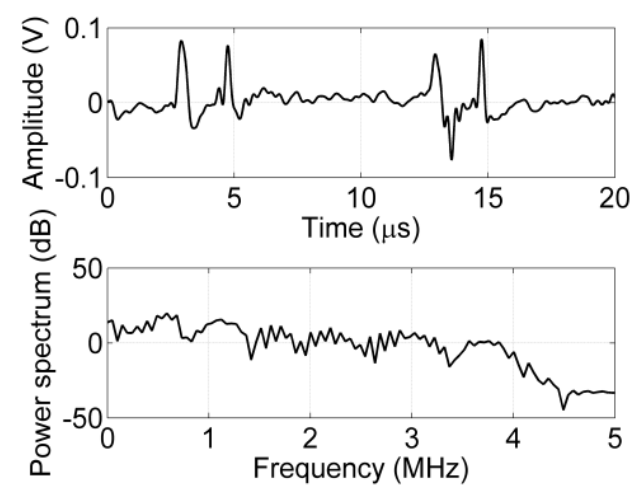

(a)

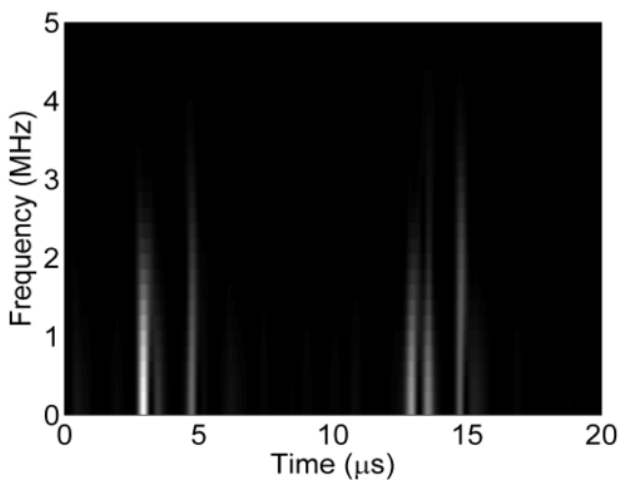

(b)

Fig. 3. (a) Normalized passive acoustic signals (upper) and its frequency spectrum of laser-induced cavitation in a DDFP droplet (below). (b) Time-frequency diagram of laser-induced cavitation in a DDFP droplet. (pulse laser energy: $45 \mu \mathrm{J}$, Temperature: $24.7^{\circ} \mathrm{C}$, with AOK-TFR).

the first pattern (a), the laser induced two small bubbles in the droplet. These two bubbles mix together and then dissipate gradually. In Figure 2(b), a single large bubble forms in the droplet and then dissipates gradually. In some cases, multiple bubbles can form when the laser is focused on the DDFP droplet, as shown in Figure 2(c). When multiple bubbles are present, they expand to their maximum radius and then dissipate with different lifespans. The data indicates that the patterns of the laserinduced cavitation in DDFP droplets are closely related to the ambient temperature. More specifically, multiple bubbles are more likely to be formed in relatively higher temperatures.

\subsection{Acoustic signal analysis}

The detected PCD signal during the LIC in the DDFP droplets and its frequency spectrum are shown in Figure 3(a). The normalized time-domain signal has four peaks. The first major peak indicates the shock wave that was emitted from the rapid expansion of the plasma. Its location along the time axis reveals the time at which the shock wave forms. Continuing, the second major peak has a similar pattern, but it is slightly smaller in amplitude. Considering the time interval of the two major 
peaks (which is always constant in all experiments), the second peak may have been caused by reflection when the travelling acoustic signal encounters a certain fixed interface: for example, the objective lens. Furthermore, it is explained that the reflection partially arrives to the hydrophone because its propagation direction is not perpendicular to the hydrophone receiving surface. The third peak may be the acoustic signal emitted by the bubble after its initial collapse, and finally, the fourth peak is the echo of peak 3 .

The spectrum analysis of the obtained acoustic signals is displayed below in Figure 3(a). The frequency domain reveals that the frequency is mainly distributed between 0 and $4 \mathrm{MHz}$. The timefrequency diagram is further shown in Figure 3(b). From the diagram, it is clear that the PCD signal has the frequency components of $0-3 \mathrm{MHz}$ at $2.91 \mu \mathrm{s}$ and $12.92 \mu \mathrm{s}$, as well as $0-3.5 \mathrm{MHz}$ at $4.75 \mu \mathrm{s}$ and $14.75 \mu \mathrm{s}$.

\section{Conclusion}

In the study, a nanosecond laser pulse with low energy was introduced in order to cause laserinduced cavitation in DDFP droplets in an in vitro experiment. Three cavitation patterns in the droplets were observed by high-speed microscopic photography, which demonstrated the close relationship of the cavitation pattern to ambient temperature. Moreover, the emitted acoustic signal was acquired and analyzed to reveal the formation process of laser-induced cavitation in DDFP droplets as well as bubble dynamics. The time-frequency analysis of the acoustic signals revealed specific frequency components at specific time points. Importantly, monitoring the acoustic signals from laser-induced cavitation in DDFP droplets is significant for the application of theranostics in microvessel damage.

\section{Acknowledgment}

Work Supported by the National Natural Science Foundation of China (Grant No. 81201105); Natural Science Basic Research Plan in Shaanxi Province of China (Program No. 2015JQ8313) and the Fundamental Research Funds for the Central Universities (No. xjj2015070).

\section{References}

[1] H. Chen, W. Kreider and A. A. Brayman, Blood vessel deformations on microsecond time scales by ultrasonic cavitation, Physical Review Letters 106 (2011), 301-304.

[2] D. Kagan, M.J. Benchimol and J.C. Claussen, Acoustic droplet vaporization and propulsion of perfluorocarbon-loaded microbullets for targeted tissue penetration and deformation, Angewandte Chemie 124 (2012), 7637-7640.

[3] X. Huang, P.K. Jain and I.H. El-Sayed, Gold nanoparticles: Interesting optical properties and recent applications in cancer diagnostics and therapy, Future Medicine 1 (2007), 681-693.

[4] G. Maatz, A. Heisterkamp and H. Lubatschowski, Chemical and physical side effects at application of ultrashort laser pulses for intrastromal refractive surgery, Journal of Optics A: Pure and Applied Optics 2 (2000), 59-62.

[5] Z.Z. Wong, O.D. Kripfgans and A. Qamar, Bubble evolution in acoustic droplet vaporization at physiological temperature via ultra-high speed imaging, Soft Matter 7 (2011), 4009-4016.

[6] P.S. Sheeran and P.A. Dayton, Phase-change contrast agents for imaging and therapy, Current Pharmaceutical Design 18 (2012), 2152-2165. 